\title{
Cordycepin augments the chemosensitivity of osteosarcoma to cisplatin by activating AMPK and suppressing the AKT signaling pathway
}

Hong-Bo Li ${ }^{1,2+}$, Jun-Kai Chen ${ }^{1,2+}$, Ze-Xin Su ${ }^{1,2+}$, Qing-Lin Jin ${ }^{1,2}$, Li-Wen Deng ${ }^{1,2}$, Gang Huang ${ }^{1,2^{*}}$ and Jing-Nan Shen ${ }^{1,2^{*}}$ (1)

\begin{abstract}
Background: Osteosarcoma is the most common primary bone tumor in children and adolescents. However, some patients with osteosarcoma develop resistance to chemotherapy, leading to a poor clinical prognosis. Hence, effective therapeutic agents that can improve the response to chemotherapy drugs to improve the prognosis of patients with osteosarcoma are urgently needed. Cordycepin has recently emerged as a promising antitumor drug candidate. This study aims to explore the effect of cordycepin in suppressing osteosarcoma in vivo and in vitro and the synergistic effect of cordycepin combined with cisplatin and to demonstrate the underlying molecular mechanism.

Methods: CCK-8 assay was performed to investigate the inhibition effect of cordycepin combined with cisplatin in osteosarcoma cell lines. The colony formation and invasion abilities were measured by colony formation assay and Transwell assay. Osteosarcoma cells apoptosis was detected by flow cytometry. Western blot analysis were used to detect the expression of cell apoptosis-related proteins and AMPK and AKT/mTOR signaling pathway-related proteins. Finally, we performed the in vivo animal model to further explore whether cordycepin and cisplatin exert synergistic antitumor effects.

Results: Notably, we found that treatment with cordycepin inhibited cell proliferation, invasion, and induced apoptosis in osteosarcoma cells in vitro and in vivo. Moreover, the combination of cordycepin and cisplatin led to marked inhibition of osteosarcoma cell proliferation and invasion and promoted osteosarcoma cell apoptosis in vitro and in vivo. Mechanistically, we demonstrated that cordycepin enhanced the sensitivity of osteosarcoma cells to cisplatin by activating AMPK and inhibiting the AKT/mTOR signaling pathway.
\end{abstract}

Conclusions: In brief, this study provides comprehensive evidence that cordycepin inhibits osteosarcoma cell growth and invasion and induces osteosarcoma cell apoptosis by activating AMPK and inhibiting the AKT/mTOR signaling pathway and enhances the sensitivity of osteosarcoma cells to cisplatin, suggesting that cordycepin is a promising treatment for osteosarcoma.

Keywords: Osteosarcoma, Cordycepin, Cisplatin, AMPK, AKT

*Correspondence: drhuanggang@126.com; shenjn@mail.sysu.edu.cn ${ }^{\dagger}$ Hong-Bo Li, Jun-Kai Chen and Ze-Xin Su, contributed equally to this work

${ }^{2}$ Guangdong Provincial Key Laboratory of Orthopedics

and Traumatology, The First Affiliated Hospital of Sun Yat-sen University, Guangzhou 510080, China

Full list of author information is available at the end of the article

\section{Background}

Osteosarcoma is the most common primary malignant bone tumor in children and adolescents and accounts for approximately $55 \%$ of all primary malignant bone tumors [1]. In the last two decades, due to the combination of surgery and chemotherapy, the 5-year 
survival rate has reached $60-70 \%$ in patients with localized tumors [2]. However, standard adjuvant/ neoadjuvant chemotherapy does not have robust antineoplastic effects in some osteosarcoma patients [3, 4], and a considerable number of patients develop drug resistance to current chemotherapy regimens [5]. Clinically, a significant number of patients are either insensitive to chemotherapy or susceptible to chemotherapy resistance under current chemotherapy regimens [5]. Therefore, novel and effective drugs for the better management of osteosarcoma patients are urgently needed.

Cordycepin, also known as 3-deoxyadenosine, a nucleoside analog, is a natural product isolated and purified from and the main active component of Cordyceps militaris. Cordycepin is a nucleoside drug and purine alkaloid that exhibits numerous biological effects, such as on anti-inflammatory Response [6], anti-immunological [7], and antitumor effects [8, 9]. Many studies have shown that cordycepin has significant antitumor effects, promoting cell apoptosis and suppressing cell proliferation and invasion and tumor metastasis through various signaling pathways [9-12]. However, the inhibitory effect of cordycepin on osteosarcoma has not been reported, and the molecular mechanisms of its anti-tumor activity remain unclear.

Cisplatin is a classic chemotherapeutic agent recommended by the National Comprehensive Cancer Network (NCCN) guidelines for the treatment of osteosarcoma via the MAP regimen [13]. Cisplatin mainly cross-links with interchain DNA in cells, resulting in DNA loss and inducing cytotoxicity [14]. Drug resistance to cisplatin, which greatly weakens the efficacy of cisplatin and prevents it from improving the prognosis of patients with osteosarcoma, occurs frequently in the clinic. Therefore, it is very important to identify effective therapeutic agents that can improve the response to chemotherapy drugs to improve the prognosis of patients with osteosarcoma.

In this study, we aimed to explore the effect of cordycepin in suppressing osteosarcoma in vivo and in vitro and to determine its antitumor molecular mechanism. In addition, we aimed to explore the synergistic effect of cordycepin and cisplatin in the treatment of osteosarcoma. The results of this study may provide evidence that cordycepin is a promising agent for the treatment of osteosarcoma.

\section{Materials and methods Reagents}

Cordycepin and cisplatin were purchased from Sigma (St. Louis, Missouri).

\section{Cell culture}

The osteosarcoma cell lines U2OS, SAOS2, MNNGHOS, 143B, MG63, SJSA1, and osteoblast cell (hFOB) were obtained from American Type Culture Collection (ATCC). The U2OS/MTX300 (U2R) cell line, a methotrexate-resistant derivative of the U2OS human osteosarcoma cell line, was kindly provided by Dr. M. Serra (Istituti Ortopedici Rizzoli, Bologna, Italy). All cell lines were cultured in Dulbecco's modified Eagle's medium (DMEM, Gibco, Grand Island, NY, USA) supplemented with $10 \%$ fetal bovine serum (Gibco, Grand Island, NY, USA), penicillin $(100 \mathrm{U} / \mathrm{mL})$ and streptomycin $(100 \mu \mathrm{g} /$ $\mathrm{mL}$ ) according to the instructions of ATCC.

\section{CCK-8 assay}

A total of 2000 osteosarcoma cells were plated in 96-well plates and exposed to different concentrations of cordycepin and/or cisplatin at $37^{\circ} \mathrm{C}$ in a $5 \% \mathrm{CO}_{2}$ humidified incubator. After the indicated times, $10 \mu \mathrm{L}$ CCK- 8 solution was added to each well, and the plates were incubated at $37^{\circ} \mathrm{C}$ for $2 \mathrm{~h}$. The absorbance was determined at $450 \mathrm{~nm}$ using a microplate reader. The proliferative inhibition rate was calculated as follows: proliferative inhibition rate $=(1-$ experimental group $/$ control group) $\times 100 \%$. The $\mathrm{IC}_{50}$ value was calculated by SPSS 20.0 software using non-linear regression analysis.

The combination index (CI) was calculated by isobologram analysis based on the Chou-Talalay method. The two drugs interactions were categorized into synergism $(\mathrm{CI}<1)$, antagonism $(\mathrm{CI}>1)$, and additive effect $(\mathrm{CI}=$ $1)$, respectively.

\section{Colony formation assay}

Cells were plated in 6-well plates in $2 \mathrm{~mL}$ DMEM containing $10 \%$ fetal bovine serum (500 cells/well). The 6-well plates were placed in a $5 \% \mathrm{CO} 2$ humidified incubator at $37^{\circ} \mathrm{C}$ and incubated with or without cordycepin and/or cisplatin. After 14days, the supernatant was removed. The cells were washed with PBS, fixed with methanol, and stained with crystal violet. After staining with crystal violet, colonies containing $>50$ cells were counted.

\section{Analysis of apoptosis by annexin V/PI staining}

Cells were plated in 6-well plates and treated with different concentrations of cordycepin and/or cisplatin for $48 \mathrm{~h}$. The cells were subsequently collected, washed with PBS twice, and resuspended in binding buffer $(500 \mu \mathrm{L})$. Then, $5 \mu \mathrm{L}$ Annexin V-FITC and $5 \mu \mathrm{L}$ PI were added to the buffer, the solution as mixed well, and the cells were 
incubated at room temperature in the dark for $15 \mathrm{~min}$. The cells were analyzed by flow cytometry.

\section{Transwell assay}

Transwell cell culture chambers with $8 \mathrm{~mm}$ microporous filter (Corning, Cat.no.353,097) with a precoating of extracellular matrix coating (BD Biosciences) were been taken out and placed in 24 well plate. $200 \mu \mathrm{L}$ of cell suspension in serum-free DMEM were add to the Transwell cell culture chambers of 24-well plates, and $500 \mu \mathrm{L}$ of DMEM with $20 \% \mathrm{FBS}$ was added to the 24 well plate. After $24 \mathrm{~h}$ of incubation at $37^{\circ} \mathrm{C}$, the cells in the Transwell cell culture chambers were stained with $0.25 \%$ crystal violet. The cells remaining in the upper Transwell chamber were removed, and those that migrated to the lower chamber were photographed and counted. Each Transwell chamber counts five fields randomly (magnification, $\times 100$ ).

\section{Western blot analysis}

Proteins were extracted from cells with RIPA buffer containing a protease inhibitor and phosphatase inhibitor (Roche), and the cell debris was removed by centrifugation $(14,000 \times g, 30 \mathrm{~min})$. The protein concentration was determined by a BCA protein assay kit. Loading buffer was added to equal amounts of protein, and the samples were boiled for $10 \mathrm{~min}$, resolved by SDS-PAGE and transferred to polyvinylidene fluoride membranes. The membranes were blocked in 5\% skim milk for $1 \mathrm{~h}$ at room temperature and incubated with a primary antibody against $\operatorname{Bax}(1: 1000$, \#5023S, CST), Bcl-2 (1:1000, \#15071S, CST), Cleaved PARP (1:1000, \#9661S, CST), MMP-2 (1:1000, \#40994S, CST), MMP-9 (1:1000, \#13667S, CST), p-AMPK (Thr172) (1:1000, \#ab133448, Abcam), AMPK (1:1000, \#ab32047, Abcam), p-AKT (Ser473) (1:2000, \#4060S, CST), AKT (1:1000, \#4685S, CST), p-mTOR (Ser2448) (1:1000, \#5536S, CST), mTOR (1:1000, \#2983S, CST), p-p70S6K (Thr389) (1:1000, \#97596S, CST), or p70S6K (1:1000, \#2708S, CST), GAPDH (1:5000, 10494-1-AP, Proteintech,) overnight at $4{ }^{\circ} \mathrm{C}$. The membranes were incubated with secondary antibodies at room temperature for $1 \mathrm{~h}$ after being washed three times with TBST. Finally, the membranes were subjected to chemiluminescence using ECL.

\section{Immunohistochemistry}

Paraffin-embedded specimens fixed in formalin were collected. The paraffin sections were placed in dimethylbenzene, dimethylbenzene, $100 \%$ ethanol, 100\% ethanol, 95\% ethanol, $90 \%$ ethanol, $80 \%$ ethanol and $70 \%$ ethanol in succession for dewaxing. After dewaxing, $3 \% \mathrm{H}_{2} \mathrm{O}_{2}$ was added for $10 \mathrm{~min}$ to block endogenous catalase activity. Citric acid buffer was added, and the sections were boiled for $3 \mathrm{~min}$, cooled to room temperature, and then boiled again. When the sections were cooled to room temperature, they were blocked with goat serum for $1 \mathrm{~h}$ at room temperature and incubated with a primary antibody (Ki-67, 1:100, \#9449, CST) overnight at $4^{\circ} \mathrm{C}$. The slides were incubated with secondary antibodies at room temperature for $1 \mathrm{~h}$ after being washed three times with PBS. Then, they were incubated with streptavidin-biotin complex $(\mathrm{SABC})$ at $37^{\circ} \mathrm{C}$ for $30 \mathrm{~min}$ and developed with $\mathrm{DAB}$ for $10 \mathrm{~min}$. Finally, the sections were counterstained with hematoxylin, dehydrated and sealed.

\section{Animal studies}

All female BALB/c nude mice (4-5weeks old) were purchased from GemPharmatech Co., Ltd. The animal experiment was approved by the Institutional Review Board of The First Affiliated Hospital of Sun Yat-sen University, and the animals were raised in the Animal Experiment Center of the First Affiliated Hospital of Sun Yat-sen University. After the mice were anesthetized with isoflurane, $1 \times 10^{6}$ suspended $143 \mathrm{~B}$ cells in $20 \mu \mathrm{L}$ were injected into the proximal tibia through the anterior tuberosity. The nude mice were randomly divided into the following four groups after approximately 14days, when the tumor volume reached approximately $200 \mathrm{~mm}^{3}$, and administered drugs by intraperitoneal (i.p.) injection: the control group, cordycepin treatment group $(40 \mathrm{mg} /$ $\mathrm{kg}$, every day), cisplatin treatment group $(5 \mathrm{mg} / \mathrm{kg}$, every 3 days), and the combined treatment group [cordycepin $(40 \mathrm{mg} / \mathrm{kg}$, every day) and cisplatin $(5 \mathrm{mg} / \mathrm{kg}$, every 3 days)]. The mice were monitored every 3 days. The size of the tumors and the weights of the mice were recorded. The size of the tumors was measured in two perpendicular dimensions (D1 and D2). The tumor volume was calculated using the formula $\mathrm{V}=4 / 3 \pi[1 / 4(\mathrm{D} 1+\mathrm{D} 2)]^{2}$, as described previously [15]. At the end of the experiment, the mice were euthanized by cervical dislocation. H\&E staining of nude mouse tissue was performed according to standard procedures.

\section{Statistical analyses}

All statistical analyses were performed using SPSS version 20.0 software. The data are expressed as the mean $\pm \mathrm{SD}$ of three independent experiments, and data from one representative experiment are shown. $P$ values were determined by Student's $t$-test or one-way ANOVA, as indicated in the legends. $P<0.05$ was considered statistically significant.

\section{Results}

Cordycepin inhibits osteosarcoma cell viability

The chemical structure of cordycepin is shown in Fig. 1a. Because cordycepin has an obvious antitumor effect 


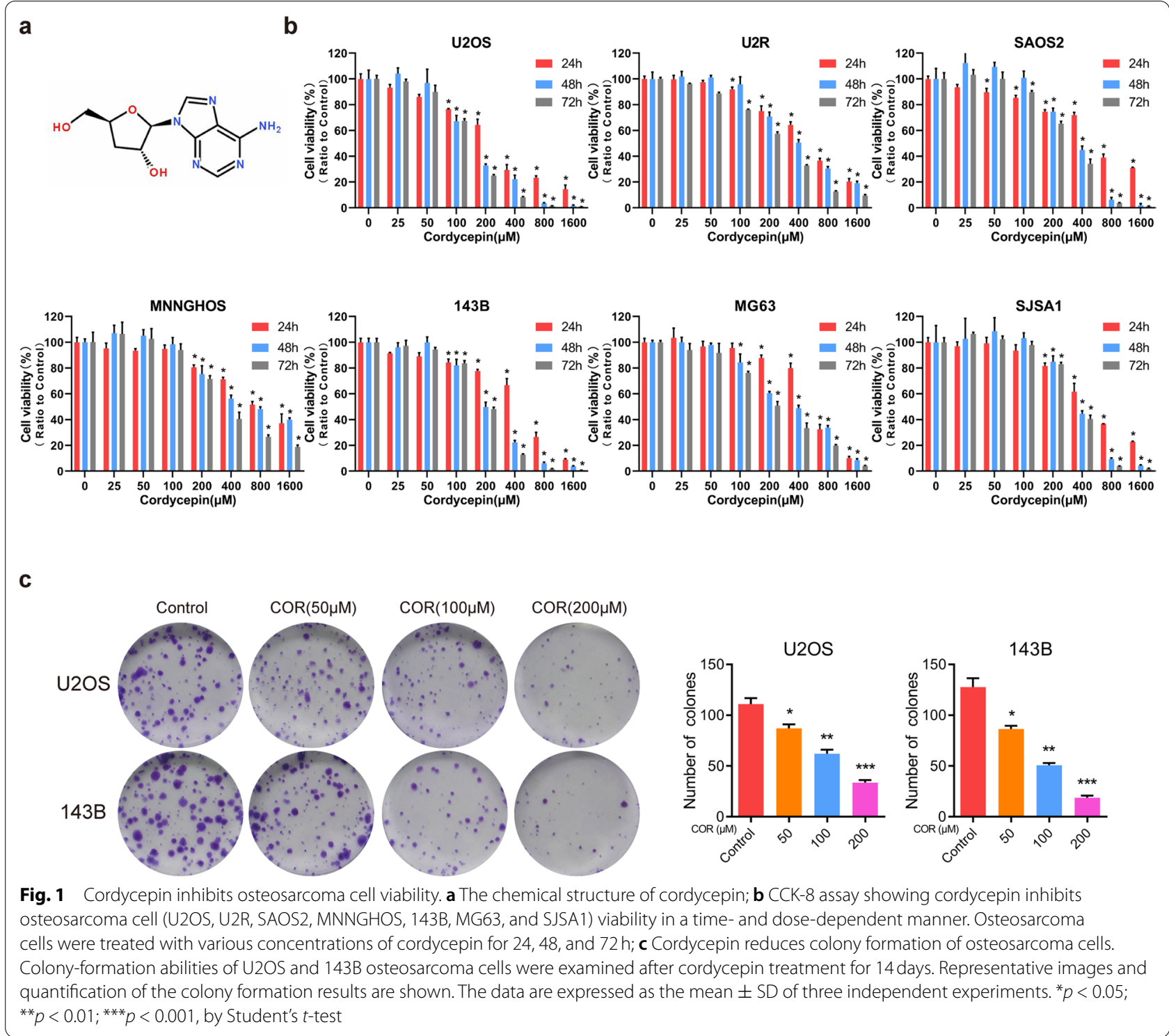

on a variety of tumors, we treated U2OS, U2R, SAOS2, MNNGHOS, 143B, MG63, and SJSA1 osteosarcoma cells with cordycepin at various concentrations for 48 or $72 \mathrm{~h}$ to investigate the toxic effect of cordycepin on these cells. The CCK- 8 assay showed that compared to control treatment, cordycepin treatment inhibited the proliferation of osteosarcoma cells, resulting in dose- and time-dependent growth inhibition (Fig. 1b). The $\mathrm{IC}_{50}$ values of cordycepin at 48 and $72 \mathrm{~h}$ were $155.1 \mu \mathrm{M}$ and $131.6 \mu \mathrm{M}$ in U2OS cells, $372.1 \mu \mathrm{M}$ and $346.3 \mu \mathrm{M}$ in SJSA1 cells, $346.0 \mu \mathrm{M}$ and $275.9 \mu \mathrm{M}$ in SAOS2 cells, $208.5 \mu \mathrm{M}$ and $191.8 \mu \mathrm{M}$ in $143 \mathrm{~B}$ cells, $443.6 \mu \mathrm{M}$ and $237.4 \mu \mathrm{M}$ in U2R cells, $775.7 \mu \mathrm{M}$ and $381 \mu \mathrm{M}$ in MNNGHOS cells, and $367.5 \mu \mathrm{M}$ and $231.6 \mu \mathrm{M}$ in MG63 cells. Additionally, we performed a colony formation assay to assess the inhibitory effect of cordycepin on U2OS and 143B osteosarcoma cells. The results revealed that treatment with cordycepin inhibited the colony formation ability of U2OS and 143B cells after incubation with cordycepin for 14 days (Fig. 1c). There was a significant dose-dependent reduction in the size and number of colony-forming cells in the cordycepin treatment group compared to the control group. These results indicate that cordycepin effectively inhibits osteosarcoma cell viability.

\section{Cordycepin induces osteosarcoma cell apoptosis}

Above, we demonstrated that cordycepin can significantly inhibit the proliferation of osteosarcoma cells. Next, we explored whether cordycepin has an effect on the apoptosis of osteosarcoma cells. We treated 
U2OS and 143B cells with different concentrations of cordycepin for $48 \mathrm{~h}$ and then collected the cells for analysis of apoptosis by Annexin V/PI staining. The results revealed that the proportion of cells labeled with Annexin V/PI in the cordycepin treatment group was increased compared to that in the control group. In contrast, cordycepin induced a limited apoptosis effect on normal osteoblast cells hFOB (Fig. 2a). Our results indicated that cordycepin significantly induced apoptosis of osteosarcoma cells in a dose-dependent manner. To further confirm these results, we measured the expression of apoptosis-related proteins in U2OS and 143B osteosarcoma cells treated with cordycepin for $48 \mathrm{~h}$ by western blotting. The results showed that the expression of the proapoptotic protein Bax was dose-dependently increased while that of the antiapoptotic protein $\mathrm{Bcl}-2$ was dose-dependently decreased after treatment with cordycepin. Furthermore, we found that the expression of Cleaved PARP was increased in the cordycepin treatment group (Fig. 2b). Therefore, our results suggest that cordycepin induces apoptosis in osteosarcoma cells.

\section{Cordycepin enhances the sensitivity of osteosarcoma cells to cisplatin to inhibit osteosarcoma cell proliferation and induce osteosarcoma cell apoptosis}

In our previous studies, we confirmed that both cordycepin and cisplatin can inhibit the proliferation of osteosarcoma cells in a dose-dependent manner. To investigate whether cordycepin can enhance the inhibitory effect of cisplatin on osteosarcoma cell proliferation, we performed a CCK-8 assay using U2OS, U2R, SAOS2, MNNGHOS, 143B, MG63, and SJSA1 cells treated with cordycepin and/or cisplatin at different concentrations for $48 \mathrm{~h}$. The results showed that the therapeutic effect of the combination of cordycepin and cisplatin was enhanced compared with that cordycepin or cisplatin alone (Fig. 3a). To determine the synergistic, additive, or antagonistic effects of combination therapy, we calculated the combination index $(\mathrm{CI})$. The results suggested that cordycepin had a synergistic effect with cisplatin (CI $<1$ ) in inhibiting osteosarcoma cell proliferation following treatment for $48 \mathrm{~h}$ (Fig. 3a). Colony formation experiments also showed that combination therapy with both cordycepin $(100 \mu \mathrm{M})$ and cisplatin $(0.5 \mu \mathrm{M})$ for 14 days inhibited the colony formation ability of U2OS and $143 \mathrm{~B}$ cells (Fig. 3b).

To explore whether cordycepin combined with cisplatin can affect the apoptosis of osteosarcoma cells, we collected cells treated with cordycepin $(200 \mu \mathrm{M})$ and/or cisplatin $(1 \mu \mathrm{M})$ at different concentrations for $48 \mathrm{~h}$ and detected cell apoptosis by Annexin V/PI staining. Apoptosis was induced in osteosarcoma cells treated with the combination of cordycepin and cisplatin compared to cells treated with cordycepin or cisplatin alone (Fig. 3c). Western blotting also showed that the expression levels of Cleaved PARP, and Bax were significantly increased by the combination of cordycepin and cisplatin compared to either drug alone. Moreover, we found that the expression of the antiapoptotic protein $\mathrm{Bcl}-2$ was significantly decreased in U2OS and 143B osteosarcoma cells treated with both cordycepin and cisplatin compared to those treated with either drug alone (Fig. 3d, e). In summary, the combination of cordycepin and cisplatin markedly promotes osteosarcoma cell apoptosis.

\section{Cordycepin increases osteosarcoma cell sensitivity to cisplatin by activating the AMPK signaling pathway and suppressing the AKT signaling pathway}

It has been reported that cordycepin may inhibit intracellular lipid accumulation through activation of AMPK via interaction with the $\gamma 1$ subunit and might act as a novel AMPK activator for the treatment of hepatic steatosis, inflammation, liver injury, and a variety of tumors by activating the AMPK signaling pathway [12, 16, 17]. To determine whether cordycepin inhibits osteosarcoma cell growth through the AMPK pathway, we performed western blotting and found that the expression of $\mathrm{p}-\mathrm{AMPK}$ was upregulated after treatment with cordycepin for $48 \mathrm{~h}$. We also found that activation of AMPK was enhanced in the group treated with the combination of cordycepin and cisplatin compared to the group treated with cordycepin or cisplatin alone (Fig. 4a, b).

The serine/threonine kinase $\mathrm{AKT}$, also known as protein kinase $\mathrm{B}(\mathrm{PKB})$, is a central node of signaling downstream of growth factors and is correlated with tumorigenicity [18]. Some reports indicate that activation of the AKT/mTOR signaling pathway plays an important role in the progression of osteosarcoma and cisplatin resistance [19-21]. To confirm whether the effect of cordycepin on the inhibition of osteosarcoma growth is mediated by suppression of the AKT/ mTOR signaling pathway, we treated osteosarcoma cells with cordycepin for $48 \mathrm{~h}$ and performed western blotting. The results verified that the expression of $\mathrm{p}-\mathrm{AKT}$, $\mathrm{p}-\mathrm{mTOR}$, and $\mathrm{p}-\mathrm{p} 70 \mathrm{~S} 6 \mathrm{~K}$ as downregulated after treatment with cordycepin. It has been reported that activation of the Akt pathway induces cisplatin resistance by inhibiting propagation of the DNA damage signal to the apoptotic machinery $[14,22]$. To further investigate whether cordycepin increases the sensitivity of osteosarcoma cells to the inhibitory effects of cisplatin by inhibiting AKT signaling, we treated osteosarcoma U2OS and $143 \mathrm{~B}$ cells with both cordycepin and cisplatin for $48 \mathrm{~h}$ and performed western blotting. The results showed that the expression levels of p-AKT, p-mTOR, and p-p70S6K were significantly decreased in the groups treated with 


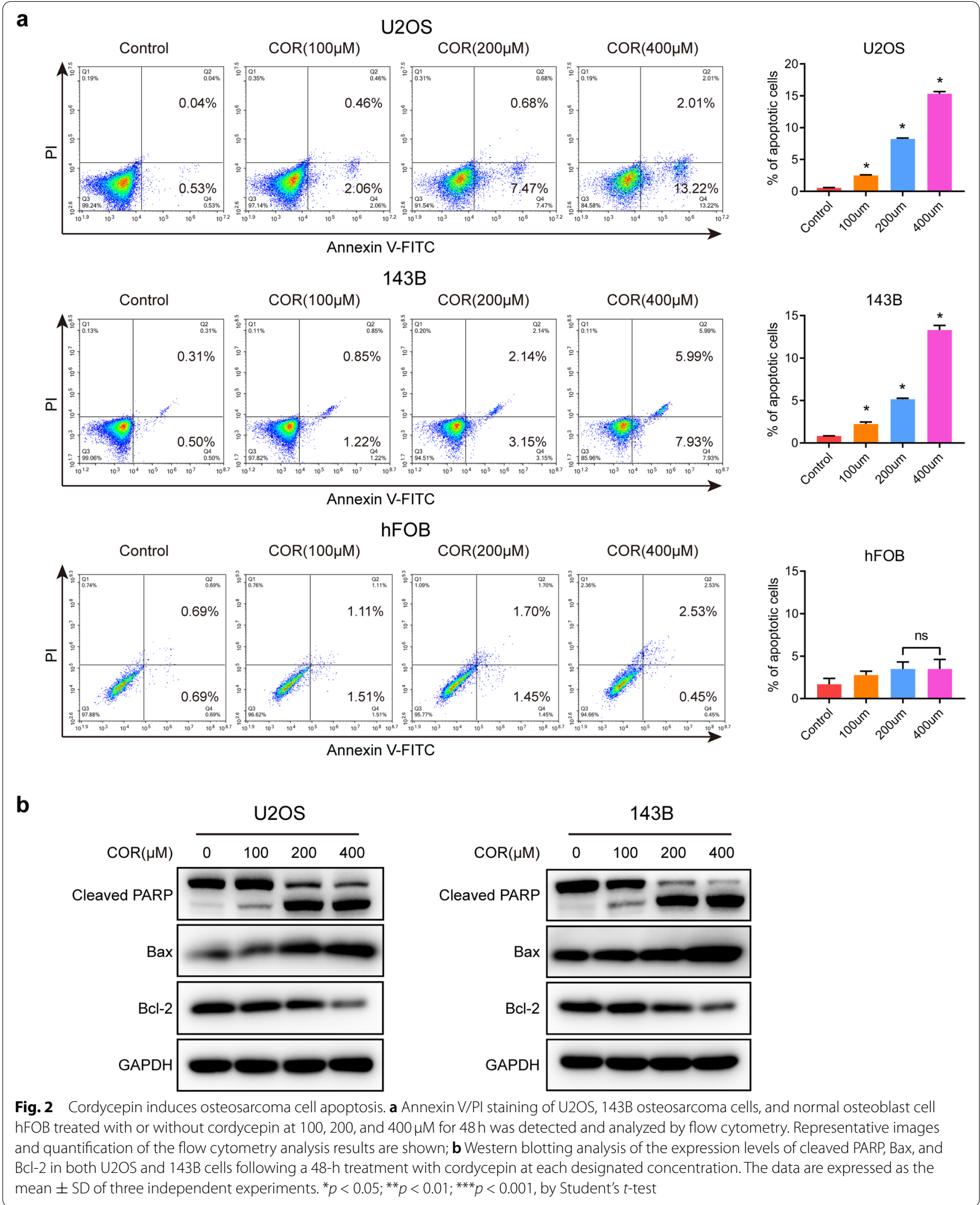


the combination of cordycepin and cisplatin compared to the control group and the groups treated with either drug alone (Fig. 4a, b). Altogether, our results indicate that cordycepin augments the chemosensitivity of osteosarcoma cells to cisplatin by activating AMPK and suppressing the AKT/mTOR signaling pathway.

\section{Cordycepin and cisplatin synergistically inhibit osteosarcoma cell invasion by downregulating MMP-2 and MMP-9 expression}

Our previous results showed that cordycepin can enhance sensitivity of osteosarcoma cells to cisplatinmediated inhibition of cell proliferation and induction of cell apoptosis. Thus, we performed Transwell assays to determine whether cordycepin enhances cisplatinmediated inhibition of osteosarcoma cell invasion. We found that cordycepin significantly reduced the cellular invasion ability of U2OS and 143B cells. Cordycepin significantly promoted the cisplatin-mediated inhibition of the cellular invasion of osteosarcoma cells (Fig. 5a). In addition, western blotting revealed that the expression levels of MMP-2 and MMP-9 were decreased in osteosarcoma cells treated with both cordycepin and cisplatin for $48 \mathrm{~h}$ compared to those in the control group and those treated with either drug alone (Fig. 5b). The above results indicate that combination therapy with both cordycepin and cisplatin synergistically inhibits osteosarcoma cell invasion.

\section{Cordycepin and cisplatin synergistically inhibit the growth of osteosarcoma xenografts in vivo}

The above experimental evidence shows that combined treatment with cordycepin and cisplatin synergistically inhibits the growth of osteosarcoma in vitro. To further explore whether cordycepin and cisplatin exert synergistic antitumor effects in vivo, we subcutaneously injected 143B cells into nude mice. When the tumor volume reached approximately $200 \mathrm{~mm}^{3}$ after ten days, the nude mice were randomly subdivided into the following four groups and administered different drugs: the control group, cordycepin treatment group $(40 \mathrm{mg}$ / $\mathrm{kg}$ ), cisplatin treatment group $(5 \mathrm{mg} / \mathrm{kg})$, and combined treatment group [cordycepin $(40 \mathrm{mg} / \mathrm{kg})$ and cisplatin $(5 \mathrm{mg} / \mathrm{kg})]$. The results showed that tumor growth was significantly reduced in the cordycepin treatment group and cisplatin treatment group compared to the control group, as reflected by tumor volume and weight (Fig. 6a-c). The growth of osteosarcoma xenografts in mice was significantly inhibited in the cordycepin $(40 \mathrm{mg} /$ $\mathrm{kg})$ and cisplatin $(5 \mathrm{mg} / \mathrm{kg})$ treatment group compared to the control group and the groups treated with either drug alone. Moreover, immunohistochemical analysis of mouse tumor samples revealed that combined treatment with cordycepin and cisplatin significantly decreased the expression of Ki-67 (Fig. 6d). In summary, cordycepin and cisplatin synergistically inhibit the growth of osteosarcoma xenografts in vivo.

\section{Discussion}

Osteosarcoma is the most common primary bone malignancy in children and adolescents, accounting for approximately $55 \%$ of all primary bone malignancies [1]. The survival rate of patients with osteosarcoma treated by surgery alone is approximately $15-17 \%$, and some osteosarcoma patients do not show significant antitumor effects after standard adjuvant/neoadjuvant chemotherapy treatment $[3,4]$. Clinically, some osteosarcoma patients are insensitive to chemotherapy or are prone to chemotherapy resistance [5]. Therefore, it is very important to identify effective therapeutic agents that can improve the response to chemotherapy drugs to improve the prognosis of patients with osteosarcoma.

Cordycepin has been reported to have significant antitumor effects, including apoptosis-inducing and cell proliferation-, invasion-, and tumor metastasis-inhibiting effects [9-12]. However, the inhibitory effect of cordycepin on osteosarcoma has not been reported. In this study, we confirmed the anti-osteosarcoma effect of cordycepin through in vitro and in vivo experiments. We demonstrated that cordycepin significantly induced apoptosis of osteosarcoma cells and significantly inhibited osteosarcoma cell proliferation and invasion. In

\footnotetext{
(See figure on next page.)

Fig. 3 Cordycepin enhances the sensitivity of osteosarcoma cells to cisplatin to inhibit osteosarcoma cell proliferation and induce osteosarcoma cell apoptosis. a CCK-8 assay showing the viability of osteosarcoma cell (U2OS, U2R, SAOS2, MNNGHOS, 143B, MG63, and SJSA1) treated with cordycepin and cisplatin at each designated concentration for $48 \mathrm{~h}$. The fraction affected (Fa)-combination index (Cl) plots showing that cordycepin exhibited an apparently synergistic effect when combined with cisplatin $(\mathrm{Cl}<1)$; b Colony formation experiments showing the colony numbers of $\mathrm{U} 2 \mathrm{OS}$ and $143 \mathrm{~B}$ cells following treatment with cordycepin $(100 \mu \mathrm{M})$, cisplatin $(0.5 \mu \mathrm{M})$, or their combination $(100 \mu \mathrm{M}$ cordycepin and $0.5 \mu \mathrm{M}$ cisplatin). Representative images and quantification of the colony formation results are shown; c Annexin V/PI staining of osteosarcoma cells (U2OS, 143B) treated with cordycepin $(200 \mu \mathrm{M})$, cisplatin $(1 \mu \mathrm{M})$, or their combination ( $200 \mu \mathrm{M}$ cordycepin and $1 \mu \mathrm{M}$ cisplatin) for $48 \mathrm{~h}$ was detected and analyzed by flow cytometry. Representative images and quantification of the flow cytometry analysis results are shown; $\mathbf{d}$, e Western blotting analysis of the expression levels of cleaved PARP, Bax, and Bcl-2 in both U2OS and 143B cells following a treatment with cordycepin (200 $\mu \mathrm{M})$, cisplatin $(1 \mu \mathrm{M})$, or their combination for $48 \mathrm{~h}$. The combination therapy of both cordycepin and cisplatin enhanced the apoptosis of osteosarcoma cells, compared to treatment with each drug alone. The data are expressed as the mean \pm SD of three independent experiments. ${ }^{*} p<0.05$; ${ }^{* *} p<0.01 ;{ }^{* *} p<0.001$, by Student's $t$-test
} 


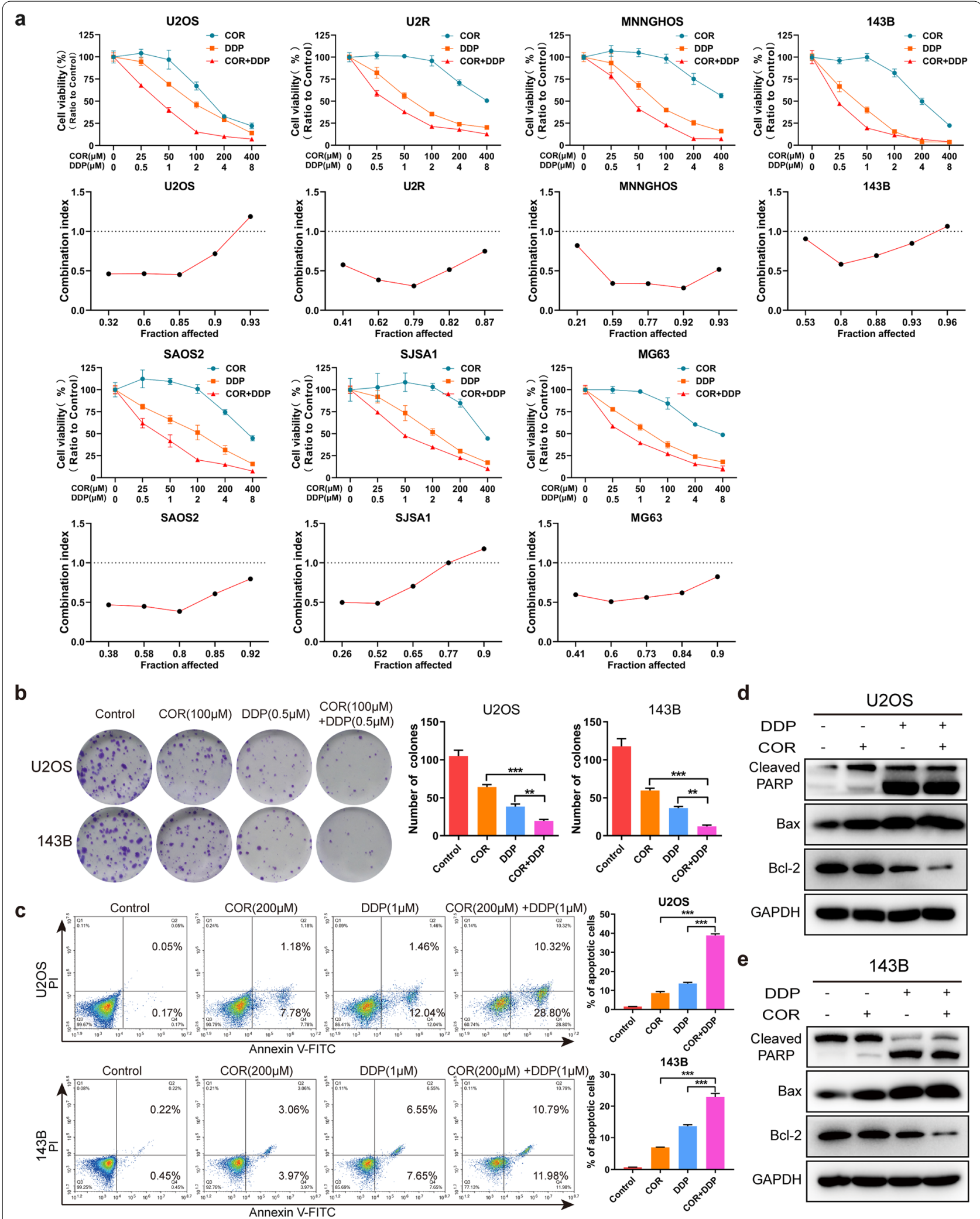

Fig. 3 (See legend on previous page.) 




addition, the inhibitory effect of cordycepin was timeand dose-dependent. We found that cordycepin significantly reduced the protein expression levels of $\mathrm{Bcl}-2$, MMP-2, and MMP-9 and induce increased the expression levels of Bax, and Cleaved PARP in osteosarcoma cells. Mechanistically, we found that cordycepin mediated the activation of AMPK and inhibited the AKT signaling pathway, thus playing a role in inhibiting the growth of osteosarcoma cells.

Cisplatin is a classic chemotherapy drug recommended by the NCCN guidelines for the treatment of osteosarcoma via the MAP regimen [13]. However, drug resistance to chemotherapy, which greatly weakens drug efficacy and prevents improvements in the prognosis of patients with osteosarcoma, often occurs during clinical use. Therefore, it is particularly important to find suitable drug combinations to improve the cytotoxicity and curative effect of chemotherapy drugs to improve the prognosis of patients with osteosarcoma. In this study, we found that cordycepin combined with cisplatin had a synergistic effect, significantly reducing resistance to cisplatin and increasing chemotherapy sensitivity. The combination of cordycepin and cisplatin showed a stronger cell-killing effect than either drug individually and reduced colony formation and cell invasion abilities. In vivo experiments also showed that the combination of cordycepin and cisplatin had a significant inhibitory effect on tumors that was more obvious than that of cordycepin or cisplatin alone, significantly reducing the tumor volume. Our study shows that cordycepin can significantly enhance sensitivity to cisplatin, providing a new therapeutic strategy for the drug treatment of osteosarcoma.

The AMPK pathway plays an important role in the regulation of cell growth and metabolism as a cellular energy regulator and regulates tumor growth and proliferation [23-25]. Cordycepin has been comfirm to inhibit drug-resistance non-small cell lung cancer progression through activating AMPK signaling pathway 


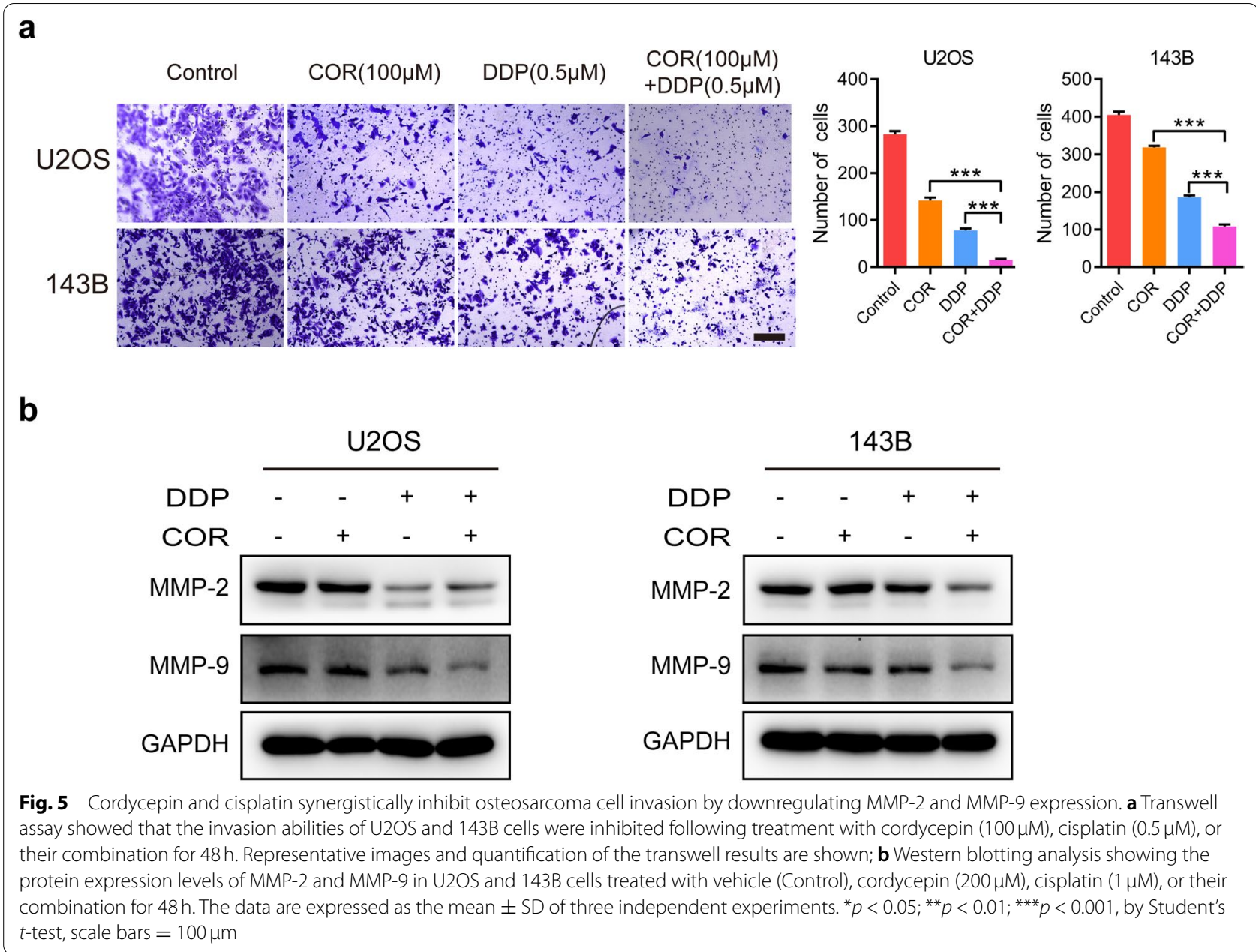

[26]. Cordycepin might be a novel AMPK activator that binds the $\alpha 1$ and $\gamma 1$ subunits near the autoinhibitory domain of AMPK $[17,27]$. Our study revealed that cordycepin mediated an increase in AMPK phosphorylation, thereby inhibiting the proliferation of osteosarcoma cells and that the expression of phosphorylated AMPK increased more significantly when cordycepin was used in combination with cisplatin than when it was administered alone. The abnormal activation of $\mathrm{AKT} / \mathrm{mTOR}$ signaling pathway can accelerate the proliferation of tumor cells, enhance resistance to apoptosis, and promote tumor invasion and metastasis [28-30]. Activation of the AKT signaling pathway is also widely believed to be associated with the resistance of tumor cells to chemotherapy [31-33]. It has been reported that activation of the AKT signaling pathway is likely to inhibit the transmission of DNA damage signals to the apoptosis machinery, resulting in the loss of DNA damage recognition and the induction of cisplatin resistance [14]. Recent studies have shown that the $\mathrm{PI} 3 \mathrm{~K} / \mathrm{AKT} / \mathrm{mTOR}$ signaling pathway is widely activated in osteosarcoma cells, especially in the drug resistant osteosarcoma cells [34]. In our study, we found that the phosphorylation levels of AKT signaling pathwayrelated proteins were decreased after treatment with cordycepin or cisplatin. More importantly, the combination of cordycepin and cisplatin had a more significant inhibitory effect on the AKT and mTOR pathways. Western blotting showed that the expression levels of p-AKT, p-mTOR and p-P70S6K were significantly decreased in the group treated with both cordycepin and cisplatin compared with the control group and the groups treated with either drug alone. P70S6K is a ribosome $40 \mathrm{~S}$ small subunit $\mathrm{S} 6$ protein kinase and an important downstream target regulated by mTOR, which plays important roles in cell proliferation, cell survival [35]. Our results showed that the combination of cordycepin and cisplatin significantly reduced the phosphorylation level of p70S6K. Emerging evidence indicates that MMPs act a pivotal part in various tumor invasion and metastasis [36]. Among them, MMP-2 and MMP-9 play a key role in initiating osteosarcoma 


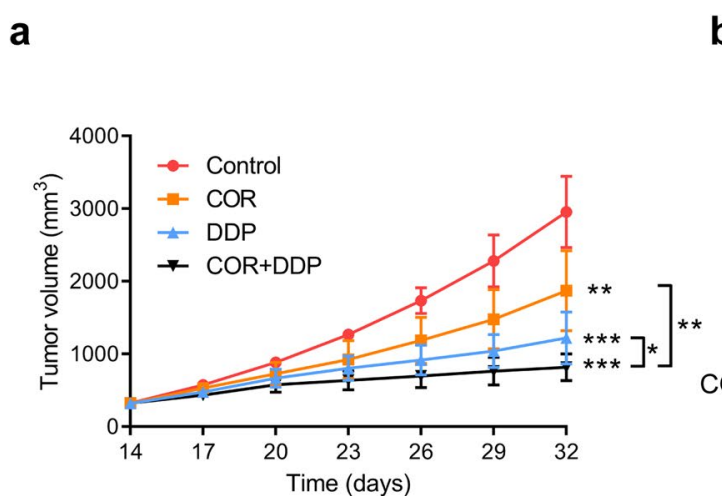

b

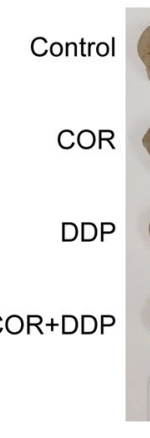

C

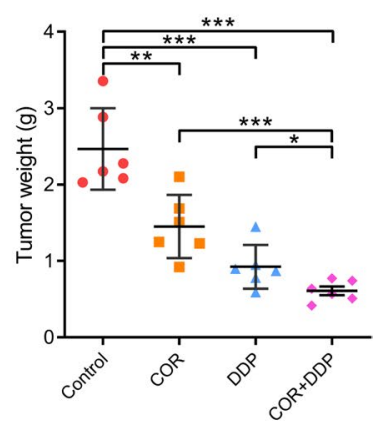

d
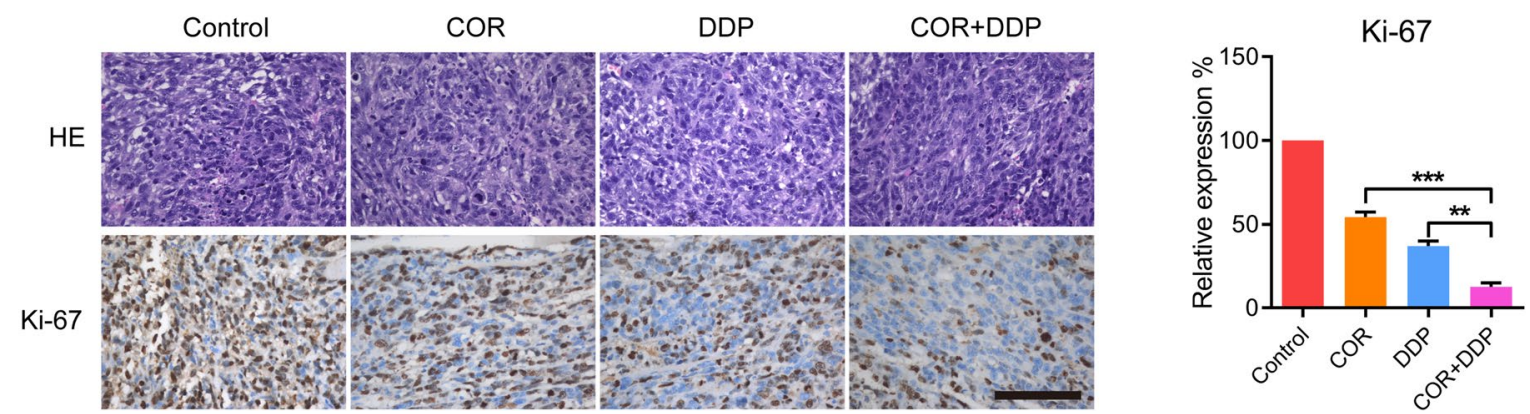

Fig. 6 Cordycepin and cisplatin synergistically inhibit the growth of osteosarcoma xenografts in vivo. a-c Examination of tumor volumes and weight to evaluate the effect of different treatments (Vehicle, cordycepin $40 \mathrm{mg} / \mathrm{kg}$, cisplatin $5 \mathrm{mg} / \mathrm{kg}$, or the combined treatment with both cordycepin $40 \mathrm{mg} / \mathrm{kg}$ and cisplatin $5 \mathrm{mg} / \mathrm{kg}$ ) on 143B cells in a xenograft model. The tumor volume of all groups was measured every 3 days. The tumor and the statistical analysis of tumor volumes in different groups was shown. Data represent the mean \pm SD of tumor volume and weight of six mice; $\mathbf{d}$ Immunohistochemistry staining of Ki-67 in mouse tumor samples to examine the inhibitory effect on osteosarcoma cell proliferation by cordycepin or/and cisplatin treatment. Combined treatment of cordycepin and cisplatin had a significant decrease in the expression of Ki-67 in vivo. The data are expressed as the mean $\pm S D$ of three independent experiments. ${ }^{*} p<0.05 ;{ }^{* *} p<0.01 ;{ }^{* *} p<0.001$, by Student's $t$-test, scale bars $=100 \mu \mathrm{m}$

cell metastasis and associate with poor response to chemotherapy in osteosarcoma [37]. Studies have shown that MMP-2 and MMP-9 are involved in AKT/ mTOR-mediated invasion and metastasis of osteosarcoma cells [38]. Our study revealed that cordycepin and cisplatin synergistically inhibited osteosarcoma cell invasion and invasion by downregulating MMP-2 and MMP-9 expression. These results indicate that cordycepin enhances the sensitivity of osteosarcoma cells to cisplatin by activating AMPK and inhibiting the AKT/mTOR signaling pathway.

\section{Conclusions}

In summary, this study provides comprehensive evidence that cordycepin inhibits the growth and apoptosis of osteosarcoma cells by activating AMPK and inhibiting the AKT/mTOR signaling pathway and enhances the sensitivity of osteosarcoma cells to cisplatin, suggesting that cordycepin is a promising novel treatment for osteosarcoma.

\section{Abbreviations}

COR: Cordycepin; DDP: Cisplatin; HE: Hematoxylin and eosin; IHC: Immunohistochemical; NCCN: National Comprehensive Cancer Network; MAP regimen: High-dose methotrexate, cisplatin, and doxorubicin..

\section{Acknowledgements}

We thank all members of the laboratory for their advice and technical assistance.

\section{Authors' contributions}

$J S, G H$ conceived and devised the study. $H L, J C, L D$ performed the in vitro and in vivo experiments and data analyses. HL, QJ, ZS wrote the manuscript. JS edited the manuscript. All authors read and approved the final manuscript.

\section{Funding}

This work was supported by the National Natural Science Foundation of China (Grant Nos. 81772860).

\section{Availability of data and materials}

All data generated or analyzed are submitted to the journal. 


\section{Declarations}

Ethics approval and consent to participate

All animal experiments were performed in accordance with a protocol approved by the ethics committee of the Institutional Animal Care of the First Affiliated Hospital of Sun Yat-sen University, China.

\section{Consent for publication}

All authors read and confirmed that this work can be published.

\section{Competing interests}

The authors declare that they have no competing interests.

\section{Author details}

'Department of Musculoskeletal Oncology, The First Affiliated Hospital of Sun Yat-sen University, Guangzhou 510080, China. ${ }^{2}$ Guangdong Provincial Key Laboratory of Orthopedics and Traumatology, The First Affiliated Hospital of Sun Yat-sen University, Guangzhou 510080, China.

Received: 2 September 2021 Accepted: 15 December 2021 Published online: 25 December 2021

\section{References}

1. Biermann JS, et al. Bone cancer. J Natl Compr Canc Netw. 2007;5(4):420-37.

2. Mirabello L, Troisi RJ, Savage SA. Osteosarcoma incidence and survival rates from 1973 to 2004: data from the surveillance, epidemiology, and end results program. Cancer. 2009;115(7):1531-43.

3. Bernthal NM, et al. Long-term results (> 25 years) of a randomized, prospective clinical trial evaluating chemotherapy in patients with highgrade, operable osteosarcoma. Cancer. 2012;118(23):5888-93.

4. Laschi $M$, et al. Establishment of four new human primary cell cultures from chemo-naive Italian osteosarcoma patients. J Cell Physiol. 2015;230(11):2718-27.

5. Bielack SS, et al. Prognostic factors in high-grade osteosarcoma of the extremities or trunk: an analysis of 1,702 patients treated on neoadjuvant cooperative osteosarcoma study group protocols. J Clin Oncol. 2002;20(3):776-90.

6. Jeong JW, et al. Anti-inflammatory effects of cordycepin via suppression of inflammatory mediators in BV2 microglial cells. Int Immunopharmacol. 2010;10(12):1580-6.

7. Li T, Wen L, Cheng B. Cordycepin alleviates hepatic lipid accumulation by inducing protective autophagy via PKA/mTOR pathway. Biochem Biophys Res Commun. 2019;516(3):632-8.

8. Baik JS, et al. Apoptotic effects of cordycepin through the extrinsic pathway and p38 MAPK activation in human glioblastoma U87MG cells. J Microbiol Biotechnol. 2016;26(2):309-14.

9. Liao Y, et al. Cordycepin induces cell cycle arrest and apoptosis by inducing DNA damage and up-regulation of p53 in leukemia cells. Cell Cycle. 2015:14(5):761-71.

10. Wong YY, et al. Cordycepin inhibits protein synthesis and cell adhesion through effects on signal transduction. J Biol Chem. 2010;285(4):2610-21.

11. Bi YM, et al. Cordycepin augments the chemosensitivity of human glioma cells to temozolomide by activating AMPK and inhibiting the AKT signaling pathway. Mol Pharm. 2018;15(11):4912-25.

12. Hawley SA, et al. Mechanism of activation of AMPK by cordycepin. Cell Chem Biol. 2020;27(2):214

13. von Mehren $M$, et al. NCCN guidelines insights: soft tissue sarcoma, version 1.2021. J Natl Compr Canc Netw. 2020;18(12):1604-12.

14. Siddik ZH. Cisplatin: mode of cytotoxic action and molecular basis of resistance. Oncogene. 2003:22(47):7265-79.

15. Berlin $\mathrm{O}$, et al. Development of a novel spontaneous metastasis model of human osteosarcoma transplanted orthotopically into bone of athymic mice. Cancer Res. 1993;53(20):4890-5.

16. Wu C, et al. Cordycepin activates AMP-activated protein kinase (AMPK) via interaction with the gamma1 subunit. J Cell Mol Med. 2014;18(2):293-304.
17. Lan T, et al. Cordycepin ameliorates nonalcoholic steatohepatitis via activation of AMP-activated protein kinase signaling pathway. Hepatology. 2021. https://doi.org/10.1002/hep.31749.

18. Manning BD, Cantley LC. AKT/PKB signaling: navigating downstream. Cell. 2007:129(7):1261-74

19. Qiu CS, et al. MNAT1 promotes proliferation and the chemo-resistance of osteosarcoma cell to cisplatin through regulating PI3K/Akt/mTOR pathway. Bmc Cancer. 2020. https://doi.org/10.1186/s12885-020-07687-3.

20. Li CC, et al. Ginsenoside Rh2 impedes proliferation and migration and induces apoptosis by regulating NF-kappa B, MAPK, and PI3K/Akt/mTOR signaling pathways in osteosarcoma cells. J Biochem Mol Toxicol. 2020. https://doi.org/10.1002/jbt.22597.

21. Xu L, et al. The inhibition of MARK2 suppresses cisplatin resistance of osteosarcoma stem cells by regulating DNA damage and repair. J Bone Oncol. 2020. https://doi.org/10.1016/j.jbo.2020.100290

22. Mitsuuchi Y, et al. The phosphatidylinositol 3-kinase/AKT signal transduction pathway plays a critical role in the expression of p21(WAF1/CIP1/ SDI1) induced by cisplatin and paclitaxel. Can Res. 2000;60(19):5390-4.

23. Mihaylova MM, Shaw RJ. The AMPK signalling pathway coordinates cell growth, autophagy and metabolism. Nat Cell Biol. 2011;13(9):1016-23.

24. Jeon SM, Chandel NS, Hay N. AMPK regulates NADPH homeostasis to promote tumour cell survival during energy stress. Nature. 2012;485(7400):661.

25. Steinberg GR, Kemp BE. AMPK in health and disease. Physiol Rev. 2009;89(3):1025-78.

26. Wei $C L$, et al. Cordycepin inhibits drug-resistance non-small cell lung cancer progression by activating AMPK signaling pathway. Pharmacol Res. 2019;144:79-89.

27. Wang $Z$, et al. Cordycepin prevents radiation ulcer by inhibiting cell senescence via NRF2 and AMPK in rodents. Nat Commun. 2019;10(1):2538.

28. Fruman DA, et al. The PI3K pathway in human disease. Cell. 2017;170(4):605-35

29. Vanhaesebroeck B, Stephens L, Hawkins P. PI3K signalling: the path to discovery and understanding. Nat Rev Mol Cell Biol. 2012;13(3):195-203.

30. Thorpe LM, Yuzugullu H, Zhao JJ. PI3K in cancer: divergent roles of isoforms, modes of activation and therapeutic targeting. Nat Rev Cancer. 2015;15(1):7-24.

31. Ediriweera MK, Tennekoon KH, Samarakoon SR. Role of the PI3K/AKT/ mTOR signaling pathway in ovarian cancer: biological and therapeutic significance. Semin Cancer Biol. 2019;59:147-60.

32. Abdul-Ghani R, et al. The PI3K inhibitor LY294002 blocks drug export from resistant colon carcinoma cells overexpressing MRP1. Oncogene. 2006;25(12):1743-52.

33. Higgins CF. Multiple molecular mechanisms for multidrug resistance transporters. Nature. 2007:446(7137):749-57.

34. Niveditha $D$, et al. Transcriptomic analysis associated with reversal of cisplatin sensitivity in drug resistant osteosarcoma cells after a drug holiday. Bmc Cancer. 2019. https://doi.org/10.1186/s12885-019-6300-2.

35. Sridharan S, Basu A. S6 kinase 2 promotes breast cancer cell survival via Akt. Cancer Res. 2011:71(7):2590-9.

36. Sato $\mathrm{H}$, et al. A matrix metalloproteinase expressed on the surface of invasive tumour cells. Nature. 1994:370(6484):61-5.

37. Kunz $\mathrm{P}$, et al. Elevated ratio of MMP2/MMP9 activity is associated with poor response to chemotherapy in osteosarcoma. BMC Cancer. 2016;16:223.

38. Lee GR, et al. Capsaicin suppresses the migration of cholangiocarcinoma cells by down-regulating matrix metalloproteinase-9 expression via the AMPK-NF-kappa B signaling pathway. Clin Exp Metastasis. 2014:31(8):897-907.

\section{Publisher's Note}

Springer Nature remains neutral with regard to jurisdictional claims in published maps and institutional affiliations. 